\title{
Conocimientos y actitudes de madres con hijos menores de cinco años sobre vacunas
}

\author{
Knowledge and attitudes of mothers with children under five years of age \\ on vaccines
}

María Arellán-Regalado ${ }^{1}$

\begin{abstract}
Resumen
Objetivo: determinar la relación entre el nivel de conocimiento y las actitudes de las madres sobre las vacunas de sus hijos menores de cinco años. Materiales y métodos: el estudio fue descriptivo y correlacional de diseño transversal. La muestra fue de 100 madres que tengan hijos menores de cinco años. En el análisis descriptivo de las variables categóricas se emplearon frecuencias y porcentajes. Para el análisis inferencial se empleó la prueba de Chi-cuadrado. Resultados: el $58 \%$ de las madres tuvo un conocimiento medio sobre las vacunas y el $51 \%$ demostró una actitud desfavorable sobre las vacunas. Las encuestadas que presentaron una actitud desfavorable tenían un nivel de conocimiento medio sobre las vacunas $(66 \%)$. Aquellas que solo estudiaban $(66 \%)$, con ingresos mayores a 1000 soles $(80 \%)$ y con primaria $(100 \%)$ presentaron un conocimiento medio sobre vacunas. Por otro lado, las madres con más de dos hijos (66\%), las empleadas informales $(100 \%)$, con ingresos mayores a 1000 soles $(80 \%)$ manifestaron una actitud desfavorable sobre las vacunas . Conclusión: las madres con un conocimiento medio presentaron una actitud desfavorable sobre las vacunas. Aquellas que solo estudian, con ingresos mayores y con primaria presentan un conocimiento medio sobre vacunas. Por otro lado, las que cuentan con un empleo, más de dos hijos e ingresos mayores manifestaron una actitud desfavorable sobre las vacunas. A partir de estos hallazgos el personal de enfermería pudiera brindar información relevante durante sus intervenciones educativas sobre inmunización a las madres.
\end{abstract}

Palabras clave: Madres; Niño; Conocimientos; Vacunas (Fuente: DeCS).

\begin{abstract}
Objective: to determine the relationship between the level of knowledge and the attitudes of mothers about vaccines of their children under five years. Materials and methods: the study was descriptive and correlational cross-sectional design. The sample was of 100 mothers who have children under the age of five. In the descriptive analysis of the categorical variables frequencies and percentages were used. For the inferential analysis, the Chi-square test was used. Results: 58\% of mothers had knowledge about vaccines and 51\% showed an unfavorable attitude about vaccines. Respondents who have an unfavorable attitude have a level of knowledge about vaccines (66\%). Those who only studied $(66 \%)$, income greater than 1000 soles $(80 \%)$ and primary $(100 \%)$. On the other hand, mothers with more than two children (66\%), informal employees (100\%), with higher incomes and 1000 soles (80\%) show an unfavorable attitude about vaccines. Conclusion: mothers with a medium knowledge presented an unfavorable attitude about vaccines. On the other hand, those who have a job, more than two children and higher incomes show an unfavorable attitude about vaccines. Based on these findings, the nursing staff could provide relevant information during the course of educational practices on immunization to mothers.
\end{abstract}

Keys words: Mothers; Boy; Knowledge; Vaccines (Source: DeCS).

Para citar:

Arellán M. Conocimientos y actitudes de madres con hijos menores de cinco años sobre vacunas. CASUS. 2018;3(3):130-137.

${ }^{1}$ Hospital Cayetano Heredia. Bachiller de Enfermería. Correo electrónico: macamar0414@g mail.com
Fecha de recepción: 20-08-18

Fecha de envío a pares: 25-08-18

Fecha de aprobación por pares : 29-11-18

Fecha de aceptación: 06-12-18 


\section{INTRODUCCIÓN}

La vacunación consiste en ejecutar todos los procesos necesarios para obtener la inmunización desde la producción de las vacunas hasta el acto físico de administrar la vacuna con previa orientación educativa sobre la misma (1). Por otro lado, la inmunización es considerada como un proceso importante de producir inmunidad mediante la administración de antígenos para la prevención y el control de las enfermedades, defunciones e incluso discapacidades principalmente en los niños menores de cinco años $(1,2)$. Del mismo modo, en el trabajo de la salud pública es considerada como una de las intervenciones de mayor éxito y rentable $(3,4)$.

\section{La Organización Panamericana de}

Salud/Organización Mundial de Salud (OPS/OMS) resalta la contribución de la inmunización en la reducción de la mortalidad infantil y menciona que esta medida de prevención evita entre 2 y 3 millones de muertes anuales a causa de la difteria, tétanos, tos ferina y sarampión $(2,5,6)$. Sin embargo, en el 2017 según la cobertura mundial de las vacunas en niños menores de cinco años se estancó y por ende solo un $86 \%$ recibieron las vacunas (2). De lo contrario si esta cobertura progresara se podrían prevenir otro 1.5 millones de muertes al nivel mundial. Con preocupación, se estima que en este año 19.5 millones de lactantes aún no reciben las vacunas básicas (2).

El Instituto Nacional de Estadística e Informática (INEI) brindó datos sobre la cobertura de vacunas específicas (BCG, pentavalente, poliomielitis $\mathrm{y}$ sarampión) con el fin de diferenciar el avance de las metas propuestas del 2012 al 2017 en el Perú. En el 2012 únicamente Apurímac contaba con el porcentaje más alto de la cobertura vacunal en menores de 36 meses de edad (85\%). Por otro lado, en el 2017 Ancash, Huánuco y Apurímac llegaron a tener una mayor cobertura (87\%) (7). Respecto a las regiones del Perú la región Sierra contó con un $64.8 \%$, la región Costa contó con una $60.1 \%$ y la región Selva con un $57.5 \%$ de cobertura vacunal (7).

El Plan Nacional Concertado del Perú 2007 - 2020 del Ministerio de Salud (MINSA), propone la atención integral de salud a la mujer y el niño privilegiando las acciones de promoción y prevención. Lo anterior con la finalidad de cumplir con el objetivo de reducir la tasa de mortalidad infantil por enfermedades prevenibles, a través de metas planeadas con el incremento de la cobertura de la vacunación en los niños menores de 5 años (8). El Perú ha ido mejorando la salud infantil en la última década, pero que aún persisten los problemas como la diarrea infantil y la necesidad de mejorar la cobertura vacunal, problemática de la morbilidad en niños y niñas (8).

Según la OMS, los objetivos de las campañas que llevan por nombre "Vaccines Work", es decir "las vacunas funcionan", buscan replicar y sensibilizar sobre la importancia de la inmunización a lo largo de la vida y prevenir muertes (4). Su finalidad es promover y brindar conocimientos e información adecuados sobre las vacunas. Un niño sin vacunas presenta mayor riesgo de a contraer enfermedades que pueden alterar su crecimiento y desarrollo (4). Sin embargo, en la actualidad existe cierta resistencia de las madres sobre la administración de las vacunas hacia sus hijos, por una dudosa posibilidad de asociación con el autismo y enfermedades del neurodesarrollo por el timerosal, compuesto que deriva del mercurio como conservante de las vacunas (6). Un estudio planteó de forma directa la relación entre la vacuna SPR (sarampión, papera y rubéola) y el autismo, pero se demostró que contaba con graves sesgos, por lo cual fue retirado. No obstante, esta información creó temor provocando la disminución de las tasas de administración de las vacunas (9). El Comité Consultivo Mundial sobre Seguridad de las Vacunas (GACVS) concluyó que no hay razón para que las vacunas sean modificadas o dejen de ser administradas (10).

Es por ello, que se debe de reconocer la responsabilidad sobre las vacunas que tiene la madre acerca del cuidado de sus hijos; ellas tomarán las decisiones y medidas beneficiosas para proteger sus vidas (6). Una madre con desconocimiento y una actitud negativa sobre las vacunas pueden ser factores suficientes para que 
un niño se encuentre expuesto a enfermedades (6, 11, 12). Las características sociodemográficas de las madres pueden asociarse con el conocimiento $(3,11)$ y actitud sobre las vacunas en sus hijos, como es el caso de la ocupación de la madre, el ingreso económico, el grado de instrucción y el número de hijos (11). Considerando la situación esbozada el objetivo del estudio fue determinar la relación entre el nivel de conocimiento y las actitudes de las madres sobre las vacunas de sus hijos menores de cinco años.

\section{MATERIALES Y MÉTODOS}

El estudio fue no experimental, descriptivo y correlacional de diseño transversal. La muestra estuvo compuesta por 100 madres que acudieron a un Centro de Salud de la ciudad de Lima para vacunar a sus hijos en el servicio de inmunización. Una característica principal de este Centro de Salud es que está ubicado en una zona rural de la ciudad de Lima donde existen niveles de pobreza elevados. El estudio fue por censo. Se incluyeron a madres de 18 a 45 años de edad con hijos menores de 5 años. No fueron considerados otros cuidadores (abuelos, hermanos, etc.) de los menores de edad.

Para evaluar la variable conocimientos de las madres sobre la vacunas de sus hijos se empleó un cuestionario validado a nivel nacional, compuesto por 18 preguntas dando valor de 2 puntos a cada ítem (13). Dicha variable fue categorizada en nivel alto (26-36 puntos), nivel medio (14-24 puntos) y nivel bajo de conocimientos (menor a 12 puntos). Por los resultados obtenidos, en el estudio se consideraron solo las categorías medio y alto. Las preguntas versaban sobre el conocimiento de los efectos adversos de las vacunas, los beneficios de aquellas que se encontraba en el calendario de vacunas, en qué caso no debería recibirlas, etc.

La variable actitudes de las madres sobre las vacunas fue categorizada como desfavorable (5-89 puntos) y favorable (90-120 puntos). La misma fue medida por una escala de Likert (desde totalmente de acuerdo hasta totalmente en desacuerdo) validada a nivel nacional, compuesta por 24 ítems. El cuestionario cuenta con preguntas referentes a la actitud sobre la importancia y beneficios de las vacunas, el esquema actual de las vacunas, las creencias, las contraindicaciones, los efectos secundarios y los refuerzos (11).

Con respecto a las variables sociodemográficas fueron: edad actual, variable categórica (18-24; 25 32; 33-45); número de hijos, variable categórica (uno, dos y más de dos); estado civil, variable categórica (casada, conviviente y soltera); ocupación, variable categórica (sin empleo, estudiante, empleada formal y empleada informal); ingreso económico, variable categórica (menos de 500 soles, de 500 a 900 soles y mayor a 1000 soles) (14); lugar de procedencia, variable categórica dicotómica (Lima y provincia) y grado de instrucción, variable categórica (primaria completa, primaria incompleta, secundaria completa, secundaria incompleta, grado superior completa e incompleta).

Al obtener los datos se analizaron utilizando el programa STATA 12, se calcularon frecuencias y porcentajes de las variables categóricas. Por otro lado, se desarrolló el análisis bivariado usando la prueba estadística Chi-cuadrado con una significancia menor o igual a un p-valor a 0.05 .

El estudio fue aprobado por un Comité de Ética y se garantizó la confidencialidad en los datos de las madres.

\section{RESULTADOS}

De las 100 madres el $58 \%$ tuvo un conocimiento medio sobre las vacunas en niños menores de 5 años y el $51 \%$ demostró una actitud desfavorable a las vacunas. Del total de las encuestadas el $54 \%$ tenía menos de 26 años, con más de 2 hijos (41\%), convivían $(86 \%)$, no tenía empleo al momento de la encuesta $(78 \%)$, contó con secundaria completa (57\%), tuvo un ingreso económico menor de 500 soles $(58 \%)$ (ver tabla 1$)$.

La variable actitud se asoció significativamente con el nivel de conocimiento sobre las vacunas $(p=0.05)$. Las encuestadas que presentaron una actitud desfavorable tenían un nivel de conocimiento medio sobre las vacunas $(66 \%)$. 
Tabla 1. Descripción de las variables de la muestra

\begin{tabular}{|c|c|c|}
\hline & $\mathbf{n}$ & $(\%)$ \\
\hline \multicolumn{3}{|l|}{ Edad } \\
\hline 18 a 26 años & 54 & 54.0 \\
\hline 27 a 35 años & 26 & 26.0 \\
\hline 36 a 45 años & 20 & 20.0 \\
\hline \multicolumn{3}{|l|}{ Número de hijos } \\
\hline Uno & 29 & 29.0 \\
\hline Dos & 30 & 30.0 \\
\hline Más de dos & 41 & 41.0 \\
\hline \multicolumn{3}{|l|}{ Estado civil } \\
\hline Casada & 11 & 11.0 \\
\hline Conviviente & 86 & 86.0 \\
\hline Soltera & 3 & 3.0 \\
\hline \multicolumn{3}{|l|}{ Ocupación } \\
\hline Sin empleo & 78 & 78.0 \\
\hline Estudiante & 5 & 5.0 \\
\hline Empleada formal & 14 & 14.0 \\
\hline Empleada informal & 3 & 3.0 \\
\hline \multicolumn{3}{|l|}{ Grado de instrucción } \\
\hline Primaria completa & 6 & 6.0 \\
\hline Secundaria completa & 57 & 57.0 \\
\hline Secundaria incompleta & 24 & 24.0 \\
\hline Grado superior completa & 7 & 7.0 \\
\hline Grado superior incompleta & 6 & 6.0 \\
\hline \multicolumn{3}{|l|}{ Ingreso económico } \\
\hline Menos de 500 soles & 58 & 58.0 \\
\hline De 500 a 1000 soles & 32 & 32.0 \\
\hline Mayor a 1000 soles & 10 & 10.0 \\
\hline \multicolumn{3}{|l|}{ Lugar de procedencia } \\
\hline Lima & 55 & 55.0 \\
\hline Provincias & 45 & 45.0 \\
\hline \multicolumn{3}{|l|}{ Conocimiento sobre vacunas } \\
\hline Medio & 58 & 58.0 \\
\hline Alto & 42 & 42.0 \\
\hline \multicolumn{3}{|l|}{ Actitudes sobre vacunas } \\
\hline Desfavorable & 51 & 51.0 \\
\hline Favorable & 49 & 49.0 \\
\hline
\end{tabular}

Mientras que aquellas con una actitud favorable presentaron a un conocimiento alto sobre vacunas (51 \%). Por otro lado, existe asociación significativa entre la ocupación de la madre y el nivel de conocimiento sobre vacunas $(\mathrm{p}=0.04)$, aquellas que solo estudiaban presentaron un conocimiento medio (100 \%). También, se encontró asociación significativa entre el ingreso económico y el nivel de conocimiento $(\mathrm{p}=0.001)$, las encuestadas con ingresos mayores a 1000 soles presentaron un conocimiento medio $(80 \%)$. Finalmente el grado de instrucción se asoció con el nivel de conocimiento $(\mathrm{p}=0.02)$, las madres con primaria completa presentaron un nivel de conocimiento medio (100\%), mientras que
Tabla 2. Asociación entre los factores sociodemográficos, las actitudes y el nivel de conocimiento sobre vacunas en madres con hijos menores de cinco años

\begin{tabular}{|c|c|c|c|}
\hline & \multicolumn{3}{|c|}{$\begin{array}{l}\text { Nivel de conocimiento } \\
\text { sobre las vacunas }\end{array}$} \\
\hline & $\begin{array}{l}\text { Medio } \\
\text { n }(\%)\end{array}$ & $\begin{array}{c}\text { Alto } \\
\text { n }(\%)\end{array}$ & $\mathbf{p}$ \\
\hline Actitudes sobre las vacunas & & & 0.05 \\
\hline Desfavorable & $34(66.6)$ & $17(33.3)$ & \\
\hline Favorable & $24(48.9)$ & $25(51.0)$ & \\
\hline Edad & & & 0.67 \\
\hline 18 a 26 años & $27(50)$ & $27(50)$ & \\
\hline 27 a 35 años & $15(57.6)$ & $11(42.3)$ & \\
\hline 36 a 45 años & $9(45)$ & $11(55)$ & \\
\hline Número de hijos & & & 0.14 \\
\hline Uno & $19(65.5)$ & $10(34.4)$ & \\
\hline Dos & $13(43.3)$ & $17(56.6)$ & \\
\hline Más de dos & $26(63.4)$ & $15(36.5)$ & \\
\hline Estado civil & & & 0.35 \\
\hline Casada & 7 (63.6) & $4(36.3)$ & \\
\hline Conviviente & $48(55.8)$ & $38(44.1)$ & \\
\hline Soltera & $3(100)$ & $0(0.0)$ & \\
\hline Ocupación & & & 0.04 \\
\hline Sin empleo & $47(60.2)$ & $31(39.7)$ & \\
\hline Estudiante & $5(100)$ & $0(0.0)$ & \\
\hline Empleada formal & $5(35.7)$ & $9(64.2)$ & \\
\hline Empleada informal & $1(33.3)$ & $2(66.6)$ & \\
\hline Ingreso económico & & & $<0.001$ \\
\hline Menos de 500 soles & $41(70.6)$ & $17(29.3)$ & \\
\hline De 500 a 1000 soles & $9(28.1)$ & $23(71)$ & \\
\hline Mayor a 1000 soles & $8(80)$ & $2(20)$ & \\
\hline Lugar de procedencia & & & 0.08 \\
\hline Lima & $28(50.9)$ & $27(49.0)$ & \\
\hline Provincias & $30(66.6)$ & $15(33.3)$ & \\
\hline Grado de instrucción & & & 0.02 \\
\hline Primaria completa & $6(100)$ & $0(0.0)$ & \\
\hline Secundaria completa & $28(49.1)$ & $29(50.8)$ & \\
\hline Secundaria incompleta & $18(75)$ & $6(25)$ & \\
\hline Grado superior completa & $4(57.1)$ & $3(42.8)$ & \\
\hline Grado superior incompleta & $2(33.3)$ & $4(66.6)$ & \\
\hline
\end{tabular}

aquellas con grado superior de instrucción presentaron un conocimiento alto (66 \%). Los demás resultados no demostraron significancia (ver tabla 2).

Se evidenció una asociación significativa entre número de hijos y las actitudes sobre vacunas $(\mathrm{p}=0.01)$, las madres multíparas (más de dos hijos) manifestaron una actitud desfavorable sobre las vacunas que reciben sus hijos (66\%), mientras que aquellas con un hijo manifestaron una actitud favorable $(68 \%)$. De igual manera, tener una ocupación se asoció con las actitudes sobre vacunas $(\mathrm{p}=0.02)$, las empleadas informales 
Tabla 3. Asociación entre los factores sociodemográficos y las actitudes sobre vacunas en madres con hijos menores de cinco años

\begin{tabular}{|c|c|c|c|}
\hline & \multicolumn{3}{|c|}{ Actitudes sobre las vacunas } \\
\hline & $\begin{array}{c}\text { Desfavorable } \\
\text { n }(\%)\end{array}$ & $\begin{array}{c}\text { Favorable } \\
\text { n }(\%)\end{array}$ & $\mathbf{p}$ \\
\hline Edad & & & 0.67 \\
\hline 18 a 26 años & $27(50)$ & $27(50)$ & \\
\hline 27 a 35 años & $15(57.6)$ & $11(42.3)$ & \\
\hline 36 a 45 años & $9(45)$ & $11(55)$ & \\
\hline Número de hijos & & & 0.01 \\
\hline Uno & $9(31.0)$ & $20(68.9)$ & \\
\hline Dos & $15(50)$ & $15(50)$ & \\
\hline Más de dos & $27(65.8)$ & $14(34.1)$ & \\
\hline Estado civil & & & 0.17 \\
\hline Casada & $5(45.4)$ & $6(54.5)$ & \\
\hline Conviviente & $46(53.4)$ & $40(46.5)$ & \\
\hline Soltera & 0.00 & $3(100)$ & \\
\hline Ocupación & & & 0.02 \\
\hline Sin empleo & $39(50)$ & $39(50)$ & \\
\hline Estudiante & $0(0.0)$ & $5(100)$ & \\
\hline Empleada formal & $9(64.2)$ & $5(35.7)$ & \\
\hline Empleada informal & $3(100)$ & 0.00 & \\
\hline Ingres o económico & & & 0.05 \\
\hline Menos de 500 soles & $31(53.4)$ & $27(46.5)$ & \\
\hline De 500 a 1000 soles & $12(37.5)$ & $20(62.5)$ & \\
\hline Mayor a 1000 soles & $8(80)$ & $2(20)$ & \\
\hline Lugar de procedencia & & & 0.43 \\
\hline Lima & $30(54.5)$ & $25(45.4)$ & \\
\hline Provincias & $21(46.6)$ & $24(53.3)$ & \\
\hline Grado de instrucción & & & 0.43 \\
\hline Primaria completa & $4(66.6)$ & $2(33.3)$ & \\
\hline Secundaria completa & $32(56.1)$ & $25(43.8)$ & \\
\hline $\begin{array}{l}\text { Secundaria } \\
\text { incompleta }\end{array}$ & $9(37.5)$ & $15(62.5)$ & \\
\hline $\begin{array}{l}\text { Grado superior } \\
\text { completa }\end{array}$ & $4(57.1)$ & $3(42.8)$ & \\
\hline $\begin{array}{l}\text { Grado superior } \\
\text { incompleta }\end{array}$ & $2(33.3)$ & $4(66.6)$ & \\
\hline
\end{tabular}

evidenciaron una actitud desfavorable (100\%). Por otro lado, las madres estudiantes encuestadas presentaron una actitud favorable (100 \%). Respecto al ingreso económico se asoció con la variable actitud sobre vacunas $(\mathrm{p}=0.05)$, las madres con ingresos mayores a 1000 soles presentaron una actitud desfavorable (80\%), mientras que aquellas que cuentan con ingresos de 500 a 1000 soles tuvieron una actitud favorable (62\%). El resto de los datos puede observarse en la tabla 3 .

\section{DISCUSIÓN}

En el estudio se determinó que el $58 \%$ de las madres que acuden al Centro de Salud tuvo un conocimiento medio sobre las vacunas de sus hijos. Asimismo, se mostró que un poco más de la mitad $(51 \%)$ de las madres encuestadas tuvo una actitud desfavorable sobre las vacunas. Además, se comprueba que existe asociación significativa entre los niveles de conocimiento y la actitud sobre la vacunas. De igual manera, se evidenció asociación entre los niveles de conocimientos de las madres sobre vacunas y la ocupación, el ingreso económico y el grado de instrucción. Por otro lado, el número de hijos, la ocupación e ingresos económicos se asociaron con la actitud sobre las vacunas.

La actitud se asocia significativamente con el nivel de conocimiento sobre las vacunas. Las madres que cuentan con un nivel de conocimiento medio de sobre la vacunas de sus hijos tienen una actitud desfavorable sobre la vacunas, mientras que tener un conocimiento alto sobre vacunas se asoció con una actitud favorable. Este resultado es corroborado por otros estudios $(6,8,11)$. En ocasiones la actitud de la madre sobre la decisión de la salud de sus hijos, en estos casos sobre las vacunas, se ve alterada por un conocimiento incompleto sobre la misma. Lo cual puede ser ocasionado por la falta información brindada por el personal de los diferentes establecimientos de salud o por adquirir informaciones erróneas obtenidas a través de fuentes no oficiales (internet) que no les permite estar seguras sobre los beneficios reales de las vacunas (6). Incluso hay madres que se sienten obligadas a vacunar a sus hijos sin conocer la importancia de las vacunas o las consideran como un mal necesario $(6,8)$. Sin embargo, las madres que cuentan con un conocimiento alto sobre las vacunas suelen tener actitudes positivas o favorables. Una madre que obtiene la información adecuada, reconoce que las vacunas son una medida de seguridad y protección para prevenir enfermedades, por ende es poco probable que las rechacen (6).

Otro hallazgo principal fue que existe asociación significativa entre la ocupación de la madre y el nivel de conocimiento sobre vacunas. Aunque las madres estudiantes presentaron solo un nivel de conocimiento medio sobre las vacunas $(15,16)$. El rol de ser madre trae consigo una serie de 
responsabilidades en el cuidado de su hijo. Sin embargo, tener otro rol como ser estudiante puede interferir de manera negativa, debido a la dificultad de compatibilizar estos dos roles pudiendo llevar a priorizar más uno de ellos (15). Por otro lado, el perfil sociodemográfico de las madres del estudio muestra que son jóvenes de bajos recursos (ganan menos de 500 soles) y viven en una zona rural alejada de la ciudad. Esto conlleva en muchos casos mayor esfuerzo y dedicación tanto para estudiar y como para cuidar a sus hijos (15). Por otro lado, existe un conflicto entre el rol en el trabajo y el rol en la familia. Este conflicto en las madres pudiera crear un ambiente de insatisfacción en el hogar sobre todo en el cuidado de los hijos y por ende una actitud desfavorable sobre las vacunas (16). Según el perfil de las madres la mayoría son jóvenes con más de dos hijos, cuenta con bajo ingreso económico, sin una adecuada educación que viven en zonas rurales. Según el INEI las zonas rurales son las más afectadas por la pobreza (17). Por lo cual, las madres consideran trabajar horas extras o tener dos trabajos para satisfacer necesidades básicas como contribuir en la canasta familiar y gastos propios del hogar esta sobrecarga pudiera contribuir a dejar de lado el cuidado y salud de sus hijos $(16,17,18)$.

Respecto al ingreso económico se asoció tanto con el conocimiento y la actitud hacia las vacunas. Las madres con ingresos superiores a 1000 soles presentaron niveles de conocimiento medio y actitud desfavorable. El ingreso económico adecuado es considerado como un factor principal para la sustentación de la familia. Por lo cual, la dedicación al trabajo de los miembros de la familia (también de la madre) es prioritaria. La inclusión de la madre al ámbito laboral, se ve como una ayuda esencial para conseguir el sustento familiar (12). Esta priorización del trabajo con el fin de obtener buenos recursos económicos pudiera poner en riesgo el nivel de conocimientos y las actitudes de la madre hacia las vacunas de sus hijos $(12,16)$.

El grado de instrucción se asoció con el nivel de conocimiento. El estudio evidencia que las madres con un grado de instrucción primaria presentaron un nivel el conocimiento medio sobre las vacunas (8). Las madres que cuentan solamente con instrucción primaria requieren más apoyo e información sobre las vacunas (8). Igualmente, las madres con un bajo nivel de educación se encuentran en un ambiente con factores de riesgo como el predominio de tabúes, prejuicios y creencias sobre las vacunas, que se convierte en una fuerte barrera que limita comprender la importancia de obtener nuevos conocimientos sobre vacunas $(19,20)$. Sin embargo, a mayor grado de instrucción de las madres, considerando que tienen más habilidades y capacidades cognitivas, tendrán mayores oportunidades de informarse; así tendrán nuevos conocimientos que les ayudarán a asumir y tomar decisiones correctas sobre el cuidado de sus hijos $(19,20)$. Las madres con solo instrucción primaria requieren por parte del personal de enfermería de más programas orientados a la promoción y prevención sobre las vacunas considerando el perfil de la población $(8$, 21). En este sentido, las sesiones educativas sobre las vacunas deben realizarse con un lenguaje sencillo de fácil compresión, con una duración adecuada, haciendo uso de técnicas pedagógicas didácticas y participativas, con el fin de que ellas movilicen sus propios recursos y les permita tomar decisiones correctas sobre la salud de sus hijos (21).

El número de hijos se asoció significativamente con la actitud hacia las vacunas. Las madres multíparas presentaron una actitud desfavorable. El aumento en el número de hijos incrementa las responsabilidades en las madres por ser las cuidadoras principales, lo que conllevaría a que no tomen un tiempo adecuado para informarse más sobre las vacunas (20). Por otro lado, la experiencia de las madres multíparas respecto a los efectos secundarios de las vacunas pudiera generar desconfianza. Lo que conlleva en algunos casos a la decisión de no volver a vacunarlos e incluso rechazan los refuerzos de las vacunas considerándolas como innecesarias (6).

Finalmente, entre las limitaciones respecto al diseño del estudio se debe considerar la presencia de sesgos de información o de memoria. 
Asimismo, algunas de las participantes del estudio no se encontraban seguras de realizar la encuesta por temor del mal uso de sus datos o vergüenza por los resultados obtenidos lo cual pudiera afectar los resultados por el sesgo de deseabilidad social. Se recomienda para futuros investigaciones sobre el tema aumentar el tamaño de la muestra, realizar levantamiento de datos no solo en un establecimiento de salud, sino en el campo o en la comunidad. Además, agregar preguntas abiertas para profundizar en las opiniones sobre las vacunas. Del mismo modo, se debería considerar información relativa a la edad de los hijos en el caso de las multíparas o el tipo de trabajo que realizan las madres y sus parejas.

\section{CONCLUSIONES}

En el Centro de Salud más de la mitad de las madres con hijos menores de cinco años de edad tienen un conocimiento medio y una actitud desfavorable sobre las vacunas. Asimismo, las madres con un conocimiento medio presentaron actitud desfavorable sobre las vacunas. Ser estudiante, presentar un ingreso económico alto y tener un grado de instrucción primaria se asociaron significativamente con el nivel de conocimiento medio. Por otro lado, tener una ocupación laboral, un ingreso económico alto y tener más de dos hijos se asociaron significativamente con la actitud desfavorable sobre las vacunas.

A partir de estos hallazgos el personal de enfermería pudiera brindar información relevante durante sus intervenciones educativas sobre inmunización en madres con los niños menores de cinco años. Asimismo, considerar de reforzar el desarrollo de los programas educativos frecuentes de manera didáctica, individualizada, con información clara, de fácil compresión. El enfermero considerando el perfil sociodemográfico deberá mantener un diálogo empático demostrando respuestas precisas y convincentes que reduzcan las dudas logrando seguridad en las madres u otros cuidadores.

\section{REFERENCIAS BIBLIOGRÁFICAS}

1. Ministerio de Salud (MINSA). Norma Técnica de Salud que establece el Esquema Nacional de 4. Organización Mundial de Salud Vacunación [Internet]. Lima, Perú: MINSA; 2018 [citado el 6 de agosto de 2018]. Disponible en: $\mathrm{ftp}: / / \mathrm{ftp} 2 . \operatorname{mins}$ a.gob.pe/normaslega les/2018/Resolucion_Ministerial_7 19-2018-MINSA1.pdf

2. Organización Mundial de Salud (OMS). Cobertura vacunal 5. Organización Panamericana de [Internet]. Ginebra, Suiza: OMS; 2018 [citado el 7 de agosto de 2018]. Disponible en: http://www.who.int/topics/immuni zation/es/.

3. Valdivia K. Conocimiento sobre inmunizaciones y su relación con factores sociodemográficos de madres con niños menores de dos años, CS “San Francisco" Tacna
2012. Artículo científico.2012:1-4. 6. Véliz L, Campos C, Vega P. Conocimiento y actitudes de los padres en relación a la vacunación de sus hijos. Revista chilena de infectología. 2016; 33(1):30-37. Inmunización [Internet]. Ginebra Suiza: OMS; 2018 [citado el 8 de agosto de 2018]. Disponible en: 7. Instituto Nacional de Estadísticas e http://www.who.int/mediacentre/e vents/2018/world-immunizationweek/es/

Salud (OPS)/ Organización Mundial de Salud (OMS). Las coberturas de vacunación para el Sarampión deben estar por encima del $95 \%$ en los niñas y niñas en la región [internet]. Ginebra, Suiza: 8. Izquierdo R. Conocimiento de OPS-OMS [citado el 8 de agosto de 2018]. Disponible en: https://www.paho.org/per/index.ph p?option=com_joomlabook\&view $=$ topic $\& \mathrm{id}=460$.
Informática (INEI). Encuesta Demográfica y de Salud Familiar 2017, Nacional y departamental [internet]. Lima, Perú: INEI; 2017 [citado el 8 de agosto de 2018]. Disponible en: https://www.inei.gob.pe/media/Me nuRecursivo/publicaciones_digital es/Est/Lib1525/index.html

madres de niños menores de un año sobre inmunizaciones y el cumplimiento del calendario vacunal. [Tesis licenciatura]. Lima: Escuela de enfermería de Padre Luis Tezza afiliada a la 
universidad Ricardo Palma; 2014. 13. Upiachihua H. Factores sociodemográficos, culturales y el cumplimiento del calendario de inmunización en lactantes. [Tesis licenciatura]. Iquitos: Universidad Nacional de la Amazonía Peruana; 2015.

2018 [internet]. Ginebra, Suiza: OMS [citado el 9 de agosto de 2018]. Disponible en: http://www.who.int/features/qa/84/ es/

10. Organización Mundial de la Salud (OMS). Declaración sobre el tiomersal. OMS [internet]. Ginebra, $\quad$ Suiza: $\quad$ OMS 2018 [citado el 10 de agosto de 2018]. Disponible en: http://www.who.int/vaccine_safety /committee/topics/thiomersal/state ment_ju12006/es/

11. Huachaca C. Conocimientos, prácticas y actitudes de las madres sobre la vacunación en sus hijos menores de 5 años, en el CS Jaime Zubieta. [Título de especialidad en enfermería]. Lima: Universidad Mayor de San Marcos; 2014.

12. Reyes R, Padrón I, Ramírez T. Trabajo y familia: ¿cómo se articula esta frágil relación? Convergencia. 2012;19(60):229253
14. ComexPerú. Sociedad de Comercio Exterior del Perú. Una mirada al APEC[internet]. Perú, Lima: Comex Perú; 2016 [citado el 13 de agosto de 2018]. Disponible en: https://www.comexperu.org.pe/me $\mathrm{dia} / \mathrm{files} / \mathrm{semanario} / \mathrm{semanario} \% 20$ comexperu\%20849.pdf

15. Sandoval J, Sepúlveda B, Amaro R, Jara M, Vergara J. Ser madre y estudiante universitaria en la Universidad de Santiago de Chile: un estudio exploratorio acerca de las implicancias psicosociales en el enfrentamiento de ambos roles. Revista de Estudios Cualitativos. 2014;1(1):23-39.

16. Padilla J, Lara B, Álvarez S. Estrés y competencia parental: un estudio con madres y padres trabajadores. Departamento de Psicología Evolutiva y de la 21. Riquelme M. Metodología de Educación. 2010;17(1):47-57.

17. CNN Español. La pobreza creció en el Perú. INEI [internet]. 2012;4(35):183-217.
España: INEI [citado el 13 de agosto de 2018]. Disponible en: https://cnnespanol.cnn.com/2018/0 4/24/pobreza-peru-por-que-inei/

18. Covarrubias M. Maternidad, trabajo y familia: reflexiones de madres-padres de familias contemporáneas. La ventana Revista de estudios de género.

19. Salgado M. Implementación de estrategia educativa para disminuir factores que influyen en el incumplimiento de vacunación en niños menores de 2 años en el centro de salud Paushiyacu. [Tesis licenciatura]. Ecuador: Universidad Regional Autónoma de los Andes;2016

20. Aguilar S, Machacca A, Vásquez D. Características sociodemográficas y culturales de madres de niños menores de un año que incumplieron el esquema de vacunación en un centro de salud. [Tesis licenciatura]. Lima: Universidad Peruana Cayetano Heredia;2017.

educación para la salud. Pediatría Atención Primaria. 2012;14 (2):77-82. 